\title{
Transtorno do Déficit de Atenção e Hiperatividade em Crianças: Uma Revisão Interdisciplinar
}

\author{
Letícia de Faria Santos ${ }^{1}$ \\ Laércia Abreu Vasconcelos \\ Universidade de Brasília
}

\begin{abstract}
RESUMO - O presente artigo revisa criticamente o amplo escopo da literatura relacionada aos critérios diagnósticos, bases etiológicas e tratamentos farmacológico e comportamental do transtorno do déficit de atenção e hiperatividade (TDAH) em crianças. Foram consultadas as bases eletrônicas MedLine, Lilacs, PsycINFO e PubMed nas últimas três décadas. Os resultados dessa revisão apontam para uma predominância do critério diagnóstico baseado no Manual Diagnóstico e Estatístico das Doenças Mentais, bem como a necessidade de uma maior interação entre variáveis biológicas e comportamentais na compreensão das bases etiológicas e de tratamento deste transtorno. Sugestões para maximizar a eficácia desta interação são apresentadas e discutidas.
\end{abstract}

Palavras-chave: transtorno do déficit de atenção e hiperatividade (TDAH); revisão; etiologia; tratamentos.

\section{Attention Deficit Hyperactivity Disorder (ADHD) in Children: An Interdisciplinary Review}

\begin{abstract}
This article critically reviews the broad scope of literature related to diagnostic criteria, the etiological basis as well as the pharmacological and behavioral treatments of the Attention Deficit Hyperactivity Disorder (ADHD) in children. The electronic databases Medline, LILACS, PsycINFO and PubMed of the last three decades were consulted. The results of this review indicated a predominance of diagnostic criteria based on the Diagnostic and Statistical Manual of Mental Disorders, and the necessity of an increased interaction between biological and behavioral variables in understanding the etiological basis and treatment of this disorder. Suggestions to maximize the effectiveness of this interaction are presented and discussed.
\end{abstract}

Keywords: attention deficit hyperactivity disorder (ADHD); review; treatments.

O Transtorno de Déficit de Atenção e Hiperatividade (TDAH) é hoje um dos temas mais estudados em crianças em idade escolar. Estima-se que ele apresente uma das principais fontes de encaminhamento de crianças ao sistema de saúde (Barkley, 2008). A compreensão conjunta das bases biológicas e comportamentais que contribuem para o desenvolvimento e manutenção dos comportamentos clássicos deste transtorno parece ser fundamental para a implementação de terapias mais eficazes. Ademais, a alta freqüência de diagnósticos de TDAH (e.g., Louzã Neto, 2010) conduz a uma reflexão crítica do processo de avaliação, intervenção, além de práticas educativas no acompanhamento de crianças e jovens tanto na família como no sistema de educação. As mudanças na família, a sofisticação do sistema de comunicação, o alto número de crianças e jovens por sala de aula constituem-se apenas em alguns dos potenciais fatores que podem contribuir para o desenvolvimento de comportamentos de risco, os quais podem ser precipitadamente classificados em diagnósticos psiquiátricos (e.g., Neves, 2008).

Embora o termo TDAH seja correntemente utilizado em contextos clínicos, acadêmicos, familiares e sociais, esta nomenclatura sofreu grandes alterações nas últimas décadas, sobretudo em função de uma melhor compreensão de suas bases etiológicas e de tratamento.

1 Endereço para correspondência: Universidade de Brasília, Instituto de Psicologia, Departamento de Processos Psicológicos Básicos, Laboratório de Análise do Comportamento. Brasília, DF, Brasil, 70910-900 E-mail: lefariasantos@gmail.com

\section{Histórico da Nomenclatura e Etiologia do TDAH}

As constantes alterações na nomenclatura e compreensão do TDAH parecem representar diferentes focos das pesquisas de cada época com suas diferentes explicações (Phelan, 2005; Rohde, Barbosa, Tramontina, \& Polanxzyk, 2000). Em 1865, as primeiras referências à hiperatividade e à desatenção não foram publicadas em literatura médica (Barkley, 2008; Rohde \& cols., 2000; Rohde \& Halpern, 2004). Apenas em 1902, a primeira descrição do transtorno foi apresentada pelos pediatras ingleses George Still e Alfred Tredgold (Barkley, 2008; Rohde \& Halpern, 2004), quais denominaram essa alteração de defeito na conduta moral acompanhado de inquietação, desatenção e dificuldades diante de regras e limites.

No início do século XX, o interesse pelo TDAH parece ter sido curiosamente despertado em decorrência de um surto de encefalite ocorrido na América do Norte entre os anos de 1917 e 1918. As crianças que sobrevieram à encefalite passavam a apresentar grande parte da sintomatologia que hoje faz parte do diagnóstico de TDAH, incluindo inquietação, desatenção e impulsividade. Embora não conclusivos e com uma terminologia marcadamente médica, diversos trabalhos foram publicados a respeito (para revisão recente, ver Barkley, 2008).

No final da década de 1930 e por todo o período da segunda guerra mundial, inúmeros casos de traumas cerebrais, acompanhados de sinais de desatenção, inquietação e impaciência, pareciam se beneficiar deste tratamento. $\mathrm{O}$ transtorno 
foi denominado de Lesão Cerebral Mínima, sendo explicado a partir de uma lesão do Sistema Nervoso Central (Benczik, 2002). Os sintomas eram semelhantes aos causados por infecção ou dano cerebral (Schwartzman, 2001). O fato de os primeiros tratamentos farmacológicos com estimulantes aliviarem o quadro encorajou diversos estudiosos na proposição de alguma relação com alterações no sistema nervoso.

Em 1962, diante da dificuldade de correlacionar os sintomas com uma determinada lesão cerebral, a Disfunção Cerebral Mínima e suas alterações comportamentais passaram a ser explicadas por disfunções em vias nervosas (Rohde \& cols., 2000; Benczik, 2002), não sendo mais apoiada em uma lesão cerebral. Em 1968, o Manual Diagnóstico e Estatístico das Doenças Mentais - DSM-II - incluiu as "desordens comportamentais da infância e adolescência" e passou nomear esse transtorno de Reação Hipercinética - níveis excessivos de atividade. O DSM-III (APA, 1980), na década de 1970, introduziu a denominação Distúrbio do Déficit de Atenção (DDA) com ou sem hiperatividade. Era consenso priorizar a desatenção, embora a hiperatividade fosse a classe de comportamentos enfatizada. Em 1987, o DSM-III-R, voltou a enfatizar a hiperatividade e alterou novamente a nomenclatura para Distúrbio de Déficit de Atenção e Hiperatividade.

Em 1993, a Classificação Internacional de Doenças CID-10 (OMS, 1993) manteve a nomenclatura do DSM-II de Transtornos Hipercinéticos. A versão revisada do DSM-IV-R ${ }^{\mathrm{TM}}$, apresentada em 1994, (APA, 2003), acrescentou aspectos cognitivos como o déficit de atenção e a falta de autocontrole ou impulsividade. De acordo com o DSM-IV$-\mathrm{R}^{\mathrm{TM}}$, a tríade sintomatológica passou a incluir desatenção, hiperatividade e impulsividade. O transtorno passou a ser denominado de Distúrbio do Déficit de Atenção/Hiperatividade - ADHD (Attention-Deficit Hiperactivity Disorder). A nomenclatura brasileira utilizada é de Transtorno de Déficit de Atenção e Hiperatividade (Barkley, 2008; Benczik, 2002).

\section{Epidemiologia}

Estudos epidemiológicos indicam que 3\% a 7\% das crianças norte-americanas com idade escolar apresentam TDAH (Goldman, Genel, Bezman, \& Slanetz, 1998; Pastor \& Reuben, 2002). No Brasil, alguns estudos em populações de crianças brasileiras em idade escolar corroboram estes índices (Guardiola, Terra, Ferreira \& Londero, 1999; Rohde \& cols., 1998; Souza, Serra, Mattos, \& Franco, 2001; Freire \& Pondé, 2005).

O transtorno apresenta uma prevalência de 9:1 de meninos para meninas, em amostras clínicas (Barkley, 2002; Rohde \& Halpern, 2004a), e uma proporção de 3:1 em amostras populacionais em geral (Offord \& cols., 1992; Barkley, 1998; Rohde \& Halpern, 2004). Vale ressaltar que os estudos epidemiológicos referem-se à faixa etária dos 7 aos 14 anos de idade (Rohde \& Mattos, 2003), embora ele possa persistir durante a vida adulta, conforme observado por Biederman e Faraone (2005). De fato, a persistência do TDAH em adolescentes e adultos vem sendo relatada em alguns estudos longitudinais, ainda que a taxa de prevalência seja inconsistente. A prevalência do TDAH em adultos na população geral varia de 2,5\% a 8\% (Kessler, Chiu, Demler,
Merikangas \& Walters, 2005; Kooij \& cols., 2005; Rohde \& cols., 1998;). De acordo Barkley (2002) e Biederman e Faraone (2005), cerca de $60 \%$ a $70 \%$ das crianças com TDAH apresentarão o diagnóstico na vida adulta.

\section{Dignóstico}

O diagnóstico do TDAH é fundamentalmente clínico (Araújo, 2002; Rohde \& cols., 2000), usualmente apoiado em critérios operacionais de sistemas classificatórios como o DSM-IV-R ${ }^{\mathrm{TM}}$ e o CID-10 (OMS, 1993), com o auxílio de exames neurológicos (Barkley \& cols., 2002; Rohde \& Halpern, 2004).

O critério do DSM-IV-R ${ }^{\mathrm{TM}}$ envolve a análise da freqüência, intensidade, amplitude (persistência em mais um contexto) e duração (pelo menos seis meses) da tríade sintomática desatenção-hiperatividade-impulsividade. A desatenção se manifesta por mudanças freqüentes de assunto, falta de atenção no discurso alheio, distração durante conversas, desatenção ou não cumprimento de regras em atividades lúdicas, alternância constante de tarefas, além de relutância no engajamento de tarefas complexas que exijam organização. A hiperatividade caracteriza-se pela fala, movimentação diurna e noturna (durante o sono) de forma excessiva, dificuldade de ficar sentado, enquanto a impulsividade envolve o agir sem pensar, mudança de atividades, dificuldade de organizar trabalhos, necessidade de supervisão e dificuldade do sujeito esperar sua vez em atividades lúdicas ou em situações de grupo. Estes sintomas devem ser acompanhados de prejuízos significativos no desenvolvimento do indivíduo (critério funcional), estar presentes em pelo menos dois (critério contextual) e ocorrer antes dos sete anos (critério temporal, um marcador não excludente). O diagnóstico deve ser refeito a cada semestre, sugerindo aspecto dinâmico e transitório do transtorno. A relação entre desatenção, hiperatividade e impulsividade, no transcorrer dos últimos seis meses, pode resultar em diferentes subtipos de diagnóstico de TDAH e a marcação temporal de seis meses parece ser importante.

De acordo com o DSM-IV-R ${ }^{\mathrm{TM}}$ (APA, 2003) três subtipos do transtorno foram definidos para o TDAH, com predomínio de: (1) desatenção, (2) hiperatividade/impulsividade e (3) combinado. Assim, é possível um diagnóstico que acuse a presença ou não de hiperatividade. O TDAH Combinado se caracteriza pela presença de seis ou mais sintomas de desatenção e seis ou mais sintomas de hiperatividade-impulsividade. A maior incidência em crianças e adolescentes é do tipo Combinado, não havendo dados acerca dos adultos (APA, 2003). O TDAH predominantemente Desatento é caracterizado por seis ou mais sintomas de desatenção e por menos de seis sintomas de hiperatividade-impulsividade. O TDAH predominantemente Hiperativo-Impulsivo deve preencher seis ou mais sintomas de hiperatividade-impulsividade e menos de seis sintomas de desatenção.

Os subtipos parecem apresentar características clínicas distintas e perfil de comorbidade. Faraone, Biedreman, Weber e Russell (1998) constataram que o subtipo TDAH Combinado apresentava maiores prejuízos funcionais do que os demais, sendo que o subtipo TDAH Desatento exige maior auxílio extraclasse, no que se refere às atividades acadêmicas. 
São prejuízos funcionais, observados no TDAH Desatento déficits de aprendizagem mais intensos, mais freqüentes nas mulheres. As crianças apresentam maior isolamento social e retraimento, somado às altas taxas de depressão e ansiedade (Rohde \& cols., 2000). O TDAH Combinado apresenta maior comprometimento nas relações sociais, reagindo de forma mais agressiva em situações aversivas e no contexto acadêmico (Millstein, Wilens, Biederman, \& Spencer, 1997; Murphy, Barkley, \& Bush, 2002). De forma semelhante, o subtipo TDAH Hiperativo/impulsivo apresenta um maior comprometimento nos relacionamentos sociais e índices mais elevados de agressividade e de transtorno de conduta (Millstein \& cols., 1997; Murphy \& cols., 2002; Souza, Serra, Mattos \& Franco, 2001).

De acordo com Rohde e Halpern (2004,) embora se observem nomenclaturas distintas, o sistema classificatório CID-10, com o Transtorno Hipercinético, e o DSM-IV-TR ${ }^{\mathrm{TM}}$, com o Transtorno de Déficit de Atenção e Hiperatividade, apresentam mais similaridades do que discordâncias nas diretrizes diagnósticas. O TDAH pode manifestar-se isoladamente apesar da alta incidência de comorbidades, isto é, a simultaneidade de ocorrência de dois ou mais transtornos ou outros problemas orgânicos (Rohde \& Benxzik, 1999). A avaliação da comorbidade é necessária no processo de implementação de tratamentos farmacológicos, considerando que este fator pode resultar em maiores perturbações comportamentais e prognósticos desfavoráveis (Biederman, Spencer \& Wilens, 2004; Rohde, Zeni, Polanczyk \& Hutz, 2004a).

Os altos índices de comorbidade no diagnóstico do TDAH, em aproximadamente $50 \%$ dos casos, envolvem riscos tanto para o diagnóstico diferencial quanto para o tratamento (Barkley, 1998; Mattos, 2005). A mais freqüente comorbidade com TDAH é o Transtorno de Conduta e Transtorno Opositor Desafiante (APA, 2003; Bierderman, Newcorn \& Sprich, 1991) com aproximadamente $30 \%$ a $50 \%$. Na população brasileira, um índice semelhante foi encontrado de 47,8\% com Transtornos Disruptivos em adolescentes (Rohde \& cols., 1999), seguidos por Transtornos de Ansiedade, em torno de 25\% (APA, 2003; Biederman \& cols., 1991; Jessen, Hinshaw \& Kraemer, 2001; Rohde \& cols., 1998; Rohde \& cols., 2004b); Depressão, com variação de 15\% a 20\% (APA, 2003; Biederman \& cols., 1991; Rohde \& cols., 1998; Jessen \& cols., 2001) e Transtorno de Aprendizagem com variação de $10 \%$ a $20 \%$. Este Transtorno inclui outros como de leitura (Dislexia), de matemática (Discalculia), e de expressão (Disgrafia) (APA, 2003; Jessen 2001; Polanchzyk \& cols., 2005; Rohde \& cols., 1998; Rohde \& cols, 2004b; Souza \& cols., 2001). Observa-se ainda em menor grau os Transtornos de Humor (APA, 2003; Jessen \& cols., 2001; Rohde \& cols, 2004b), de personalidade (Barkley, 1998), a Enurese, além de tiques (Biederman, Wilens, Mick, Spencer \& Faraone, 1999).

\section{Etiologia}

O TDAH parece resultar de uma combinação complexa de fatores genéticos, biológicos, ambientais e sociais. Ao destacarem fatores genéticos no TDAH, alguns estudos indicam marcadores fenotípicos familiares (Todd, 2000), bem como marcadores genéticos (Biederman \& Faraone, 2005; Faraone \& cols., 2005; Rohde \& cols., 2004a) sendo a transmissão poligenética também considerada (Rohde \& Halpern, 2004; Todd, 2000; Rohde \& cols., 2004a; Thapar, Holmes, Poulton \& Harreington, 1999). O fator biológico da transmissão do TDAH tem sido demonstrado em estudos comparativos de gêmeos monozigóticos e dizigóticos, que não residem em uma mesma cidade, e filhos adotivos (Kazdin \& Kagan, 1994; Johnston \& Mash, 2001; Michelson \& cols., 2001; Rutter \& Sroufe, 2000). Recorrências familiares têm revelado um alto índice de influência hereditária, de 25,1\% a 95\% (Biederman, Faraone, Keenan, Knee \& Tsuang, 1990; Biederman \& Faraone, 2005; Faraone \& cols., 2005; Thapar \& cols., 1999), contra 4,6\% da população geral. Vale ressaltar que os estudos genéticos envolvendo TDAH não excluem as influências culturais, familiares e exposições a eventos estressantes (Biederman \& Faraone, 2005; Rotta, 2006; Rohde \& Halpern, 2004).

Alterações nos substratos neurais que regulam as funções executivas também têm sido consideradas na etiologia do TDAH (Szobot \& Stone, 2003; Barkley, 1997; Faraone \& cols., 1998; Barkley, 2008). Segundo o modelo Barkley (1997), a desatenção no TDAH deriva do mau funcionamento das funções executivas, caracterizando-se principalmente por uma dificuldade em inibir comportamentos e de controlar as interferências. As conseqüências da falha neste processo inibitório seriam responsáveis pelas sintomatologias de baixa tolerância à espera, alta necessidade de recompensa imediata, falta de um comportamento governado por regras, falha na previsão das conseqüências e emissão de respostas rápidas, porém imprecisas (Barkley e cols., 2008, Strayhorn, 2002). Estas sugestões têm sido corroboradas por estudos de neuroimagem (Barkley, 2002; Guardiola \& cols., 1999) e por modelos cognitivos e neuropsicológicos (Satterfield \& Dawson, 1971; Sonuga-Barke, 2002; Szobot \& Stone, 2003).

Do ponto de vista neuroquímico, parece haver consenso da participação predominante da dopamina e da noradrenalina, sobretudo pelo fato das principais drogas utilizadas no tratamento do TDAH atuarem como agonistas indiretos desses neurotransmissores, a exemplo dos psicoestimulantes da classe das anfetaminas (p.ex., metilfenidato. O reconhecido papel exercido pela noradrenalina na atenção e da dopamina nos centros motores reforçam essa idéia.

\section{Tratamentos e Diretrizes Futuras}

O tratamento do TDAH requer uma abordagem múltipla, englobando intervenções psicoterápicas e farmacológicas (Anastopoulos, Rhoads \& Farley, 2008) com a participação de múltiplos agentes sociais como pais, outros familiares, educadores, profissionais de saúde, além da própria criança.

Três tipos de tratamento do TDAH têm sido empregados: farmacológico, terapia comportamental e a combinação das terapias farmacológica e comportamental (Swanson \& cols., 2001), sendo este último considerado como a forma mais eficaz (Jessen, 2001).

Nos anos 1930, pesquisas mostraram que drogas estimulantes como o Metilfenidato e Pemoline aumentavam o nível de catecolaminas no cérebro, normalizando temporariamente 
os comportamentos clássicos do TDAH (Smith \& Strick, 2001; Zametkin \& Rapoport, 1987). Segundo Barkley e cols. (2008), a adição de fármacos estimulantes promove uma amenização dos sintomas motores, impulsividade e desatenção e uma elevação das interações sociais e desempenho acadêmico.

A compreensão do princípio ativo do Metilfenidato, fármaco freqüentemente utilizado no tratamento do TDAH é o principal suporte para a teoria da hipofunção dopaminérgica, por promover um aumento de dopamina na fenda sináptica em regiões como o striatum (Biederman \& Spencer, 1999; Thapar \& cols., 1999; Vaidya e cols., 1998) e, conseqüentemente, uma diminuição dos efeitos característicos do TDAH. Contudo, o envolvimento destes neurotransmissores parece ser bem mais complexo, sendo sensíveis aos tipos de receptores envolvidos e às regiões cerebrais envolvidas (Biederman \& Spencer, 1999).

Outros fármacos com comprovada ação noradrenérgica têm se mostrado eficazes no tratamento do TDAH, apoiando a hipótese noradrenérgica na modulação do Transtorno (Bierdeman \& Spencer, 1999). Portanto, tratamentos farmacológicos, especialmente com base na administração de substâncias psicoestimulantes, como o Metilfenidato e Pemoline, têm se mostrado úteis, sendo os fármacos mais utilizados no tratamento do TDAH. Os antidepressivos tricíclicos (Imipramina, Desipramina, Amitriptilina, Clomipramina), agonistas de receptores do tipo a2 (Clonidina, Guanfacina), agonista de noradrenalina e Atomoxetina, Modafinil e Bupropiona também são utilizados no tratamento de TDAH embora não sejam as medicações de primeira linha (Biederman \& Faraone, 2005; Guardiola \& cols, 1999; Segenreich e Mattos, 2004).

Nos Estados Unidos, os estimulantes aprovados pela U.S. Food and Drug Administration (FDA) são: o Metilfenidato, (composto de sais de sulfato de anfetamina e dextroanfetamina), a Pemoline de magnésio e as anfetaminas (Rotta, 2006; Correia Filho \& Pastura, 2003). No Brasil, o único psicoestimulante disponível é o Metilfenidato, (Ritalina ${ }^{\circledR}$ e Concerta $\left.{ }^{\circledR}\right)$ com duas formas de ação, curta e longa. A medicação de curta duração é comercializada com o nome de Ritalina ${ }^{\circledR}$, na apresentação convencional de $10 \mathrm{mg}$, com uma duração de 3 a 4 horas. A Ritalina ${ }^{\circledR}$ tem sistema de liberação em dois pulsos, mimetizando o esquema do Metilfenidato de curta-ação quando administrado duas vezes ao dia (Correia Filho \& Pastura, 2003; Rotta, 2006). Entretanto, a Ritalina LA ${ }^{\circledR}$, possui três apresentações $20 \mathrm{mg}, 30 \mathrm{mg}$ e 40 mg, com duração de 6 a 8 horas, sendo comum apenas uma administração diária. A eficácia de ambas as formulações é similar (Wilson, Cox, Merkel, Moore \& Coghill, 2006) Desta forma, a Ritalina LA ${ }$ se diferencia da Ritalina ${ }^{\circledR}$ pelo tempo de ação.

Com um tempo de ação superior ao da Ritalina LA ${ }^{\circledR}$, o Concerta $\AA$, com apresentações de $18 \mathrm{mg}, 36 \mathrm{mg}$ e 54 mg, possui tempo de ação de 10 a 12 horas. Seu sistema de liberação osmótica oral (OROS) permite uma liberação constante, evitando variações de concentração plasmática. Os medicamentos de uso prolongado apresentam vantagens no que se refere à manutenção dos efeitos terapêuticos ao longo do dia e diminuição dos efeitos colaterais (Heger \& cols. 2006), além de favorecer a adesão terapêutica e evitar o uso da medicação na escola (Correia Filho \& Pastura, 2003; Heger \& cols. 2006; Rotta, 2006).

A eficácia do uso destes psicoestimulantes no tratamento do TDAH tem sido sustentada por dados que indicam melhora no desempenho em teste de tempo de reação e de atenção concentrada, bem como no teste clínico da onda P300 (Klorman, 1991; Lousier, McGranth, \& Klein, 1996; Sonneville, Njiokikjien \& Bos, 1994; Sykes, Dougkas \& Morgentersn, 1972; Van der Meere, Boudewijn, \& Stemerdink, 1996).

A medicação tem sido utilizada nos períodos escolares, sendo comumente suspensa aos finais de semana e férias. A interrupção visa amenizar os efeitos colaterais de longo prazo e, em curto prazo, os efeitos secundários. Entre os efeitos de curto prazo mais freqüentes, observa-se a redução de apetite, anorexia, insônia, ansiedade, irritabilidade, labilidade emocional, cefaléia e dores abdominais. Com menor freqüência verificam-se alterações de humor, tiques, pesadelos e isolamento social (Barkley, McMurray, Edelbrock \& Robbins, 1990; Rohde \& Mattos, 2003; Wilens \& cols., 2003). Com a freqüência mais baixa, contudo, envolvendo alta periculosidade, verificou-se a psicose como efeito do uso do Metilfenidato (Schteinschnaider \& cols., 2000).

No sistema de educação brasileiro não é incomum a presença de professores em sala de aula diagnosticando de maneira intuitiva os estudantes que apresentam padrões de comportamentos que perpassam o TDAH. Essas crianças são fonte de medo e insegurança por parte dos educadores por não terem uma ampla visão de desenvolvimento ou de estratégias pedagógicas que favorecem a aprendizagem daqueles que se mostram diferentes ou que desafiam uma rotina escolar.

A despeito da grande eficácia anunciada do tratamento farmacológico (e.g. Bierdeman \& Spencer, 1999, Van der Meere, Boudewijn, \& Stemerdink, 1996), o tratamento psicoterápico tem se mostrado útil ao trabalhar com todo o contexto social da criança diagnosticada (pais e professores). Toda e qualquer intervensão psicológica é realizada no campo das relações do organismo com o ambiente. O terapeuta fornecerá informações sobre o TDAH, promovendo alterações ambientais que favoreçam o desenvolvimento da criança. A interação terapeuta-pais voltada para as práticas educativas é um dos alvos com os resultados mais positivos (Rohde \& Halpern, 2004), auxiliando na alteração de práticas educativas aversivas (Benczik, 2002). A educação sobre o transtorno para as crianças, pais e professores constitui uma parte fundamental das terapias comportamental e ou cognitivas.

A análise do comportamento, além de promover uma intervenção psicoeducativa, contribui de forma significativa, para o estudo desta síndrome ao demonstrar que manipulações nas variáveis ambientais podem promover alterações nos padrões comportamentais de impulsividade, hiperatividade e desatenção (Bernado, 2004). A identificação destas variáveis contribui para desfazer rótulos prévios que freqüentemente acompanham as crianças e que não favorecem um engajamento em novas contingências e o desenvolvimento de novos padrões comportamentais. Baseado na análise funcional e histórica dos comportamentos apresentados pela criança com TDAH, diversas técnicas têm se mostrado úteis. O sistema de pontos, reforçamento diferencial, custo da resposta, tarefas para casa, modelação e dramatização tem sido as mais utilizadas (Knapp, Johannpeter, Lyszkowski \& Rohde, 2002). 
A terapia cognitiva apoia-se na compreensão de que o TDAH é função de um quadro de deficiência de estratégias cognitivas. Para tal, prioriza o seu desenvolvimento por meio de intervenções como: auto-instrução, registro de pensamentos disfuncionais, solução de problemas, auto-monitoramento, auto-avaliação e planejamento e cronogramas.

Considerando a complexidade das diversas variáveis determinantes do TDAH, o atendimento de crianças com este diagnóstico tem sido feito preferencialmente por equipes multidisciplinares, sendo importante ressaltar que as concepções de desenvolvimento influenciam a avaliação e intervenção (Bijou \& Baer, 1978; Bijou \& Baer, 1978b). Ao considerar este ponto, vale ressaltar que os analistas do comportamento não adotam teoria do desenvolvimento, para estes, o desenvolvimento humano é resultado de interações dinâmicas e bidirecionais entre o indivíduo ativo e o ambiente físico e social, incluindo a família, escola e os centros de saúde. Desta forma, a ação do organismo altera aspecto do ambiente, os quais retroagem sobre as ações do organismo (Skinner, 1957/1978).

$\mathrm{Na}$ análise do comportamento, o conjunto de todas as interações do organismo e seu ambiente irá compor a história de desenvolvimento, sendo essa ideográfica. Contudo, este conjunto de mudanças nas interações organismo-ambiente promove relações funcionais que podem ser favoráveis ou de risco, para o organismo e seu ambiente (Rosales-Ruiz \& Baer, 1996). A exemplo, crianças com diagnóstico TDAH apresentam uma história com múltiplas interações bidirecionais entre o organismo e ambientes físico e social o qual reforçou (produziu) e mantém um padrão comportamental de desatenção, impulsividade e/ou hiperatividade.

Um trabalho interdisciplinar entre a neurociência e os analistas do comportamento pode favorecer intervenções clínicas construindo novos padrões de atenção, autocontrole e engajamento em atividades de alto custo, favorecendo a adaptação do sujeito com diagnóstico de TDAH em seu ambiente social, escolar e familiar. Kennedy, Caruso e Thompson (2001) afirmam que os conhecimentos desenvolvidos pela neurociência podem favorecer os analistas do comportamento na aplicação de seus conhecimentos. De forma similar, a neurociência pode ser beneficiada com alguns dados desenvolvidos pelos analistas do comportamento que demonstram o efeito de variáveis ambientais, como a atenção social no ambiente escolar produzindo a atenuação dos sintomas (Kodak, Northup \& Kelley, 2007) ou o controle verbal sobre os comportamentos classificados como inapropriados (Falcomata \& cols., 2008).

A abordagem funcional que direciona toda análise clínica desenvolvida por analistas do comportamento também é fundamental para o diagnóstico do transtorno de TDAH. Uma vez que este é essencialmente clínico (Araújo, 2002; Rohde \& cols., 2000), baseado em entrevistas com a criança e parentes. A entrevista que subsidia a análise clínica do médico irá se apoiar em descrições comportamentais das crianças, em diversos contextos e o impacto destes comportamentos nos contextos familiar, escolar e social em geral.

Futuras pesquisas que envolvam as relações entre os comportamentos, práticas educativas, o universo midiático e arranjos familiares que mantém os clássicos padrões de comportamentos característicos do TDAH podem enriquecer sobremaneira a compreensão das influências de determinadas contingências comportamentais e metacontingências presentes na história e manutenção do Transtorno de Déficit de Atenção/Hiperatividade. Nas trocas interdisciplinares entre a psicologia e a medicina, ressalta-se que o diagnóstico psiquiátrico no contexto contemporâneo é caracterizado por um "diagnóstico continuado", a partir de várias sessões de observação e de trocas interdisciplinares entre a psiquiatra e a psicologia, no transcorrer do processo de desenvolvimento de crianças e jovens (Ciasca, 1994). Há riscos em posições extremas do continuum de diagnóstico - do nunca diagnosticar ao sempre diagnosticar. O equilibro neste continuum dependerá de trocas efetivas entre profissionais de saúde, educação, além da família visando a promoção da sofisticação do repertório comportamental das crianças e jovens, para além de uma visão linear de eliminação de comportamentos inapropriados (e.g., Goldiamond, 2002).

\section{Referências}

American Psychiatric Association. (1980). Diagnostic and statistical manual of mental disorders. (3rd ed.). Washington: American Psychiatric Association.

American Psychiatric Association. (2003). Manual diagnóstico e estatístico de transtornos mentais ( $4^{\circ} \mathrm{Ed}$.) (Dornelles, C. trad.). (primeira publicação 2000) Porto Alegre: Artmed.

Anastopoulos, A. D., Rhoads, L. H., \& Farley, 2008) Aconselhamento e Treinamento de para os Pais. Em R. A. Barkley, e cols., (Orgs.), Transtorno de Déficit de Atenção/Hiperatividade Manual para diagnóstico e Tratamento. Artemed: $3^{\circ}$ Edição.

Araújo, A. P. Q. C. (2002). Avaliação e manejo de crianças com dificuldade escolar e distúrbio de atenção. Jornal de Pediatria; 78 (supl.1), S104 - S110.

Barkley, R. (1998). Attention-deficit hyperactivity disorder: a handbook of diagnosis and treatment, New York: Guilford.

Barkley, R. A. (1997). Behavioral inhibition, sustained attention, and executive functions: Constructing a unifying theory of ADHD. Psychological Bulletin, 121, 65-94.

Barkley, R. A. (2002). Major life activity and health outcomes associated with Attention-Deficit/Hyperactivity Disorder. Journal of Clinical Psychiatry, 63, 10-15.

Barkley, R. A. (2006). Driving risks in adults with ADHD: Yet more evidence and a personal story. The ADHD Report, 14(5), 1-10.

Barkley, R. A., Anastopoulos, A. D., Robin, A. L., Lovett, B. J., Smith, B. H., Cunningham, C. E., Shapiro, C. J., Connor, D. F., DuPaul, G. J., Prince, J. B., Dooling-Litfin, J. K., Biederman, J., Murphy, K. R., Rhoads, L. H., Cunningh, L. J., Pfiffner, L. J., Gordon, M., Farley, S. E., Wilens, T. E., Spencer, T. J., Hathway, W. (2008). Transtorno de déficit de atenção/hiperatividade: manual para diagnostico e tratamento ( $3^{\circ} \mathrm{ed}$.). Porto Alegre: Artmed.

Barkley, R. A., McMurray, M. B., Edelbrock, C. S., \& Robbins, K. (1990). Side effects of metlyiphenidate in children with attention deficit hyperactivity disorder: a systemic, placebo-controlled evaluation. Pediatrics, 86(2), 184-192.

Benczik, E. B. P. (2002). Transtorno de déficit de atenção/ hiperatividade - Atualização diagnóstica e terapêutica. São Paulo: Casa do Psicólogo. 
Bernado, P. C. (2004). Autocontrole de crianças diagnosticadas com TDAH: o efeito de atrasos absolutos e relativos em escolh a com tentativas discretas. Dissertação de Mestrado, Universidade de Brasília, Brasília.

Biederman, J., \& Faraone, S. (2005). Attention-deficit hyperactivity disorder. Lancet, 366, 237-248.

Biederman, J., \& Spencer, T. (1999). Attention-deficit/hyperactivity disorder (adhd) as a noradrenergic disorder. Biological Psychiatry, 46, 1234-1242.

Biederman, J. (1998). Attention-deficit/hyperactivity disorder: A life span perspective. Journal of Clinical Psychiatry, 59(7), 4-16.

Biederman, J., Newcorn, J., \& Sprich, S. (1991). Comorbidity of Attention-Defict/Hyperactivity Disorder with Conduct, Depressive, Anxiety, and Other Disorders. American Journal Paychiatry, 148, 564-577.

Biederman, J., Faraone, S. V., Keenan, K., Knee, D., \& Tsuang, M. T. (1990). Family-genetic and psychosocial risk factors in DSM-III attention deficit disorder, Journal of the American Academy of Child and Adolescent Psychiatry, 29(4), 526-533.

Biederman, J., Spencer, T. \& Wilens, T. (2004). Evidence-based pharmacotherapy for attention-deficit hyperactivity disorder. The International Journal of Neuropsychopharmacology, 7(1), 77-97.

Biederman, J., Wilens, T., Mick, E., Spencer, T., \& Faraone, S. V. (1999). Pharmacotherapy of attention-deficit/hyperactivity disorder reduces risk for substance use disorder. Pediatrics, 104(2), 1-5.

Bijou, S. W., \& Beer, D. M. (1978b). Psicología del desarrollo infantil e teoria empírica y sistemática de la conducta. Editora Trillas: Mexico, vol 1.

Bijou, S. W., \& Baer, D. M. (1978). Behavior analysis of child development. Englewood Cliffs, NJ: Prentice-Hall.

Ciasca, S. M. (1994) Distúrbios e dificuldades de aprendizagem em crianças: análise do diagnóstico interdisciplinar. Tese de Doutodo, Faculdade de Ciências Médicas da Unicamp, Campinas, Brasil.

Correia Filho, A.G., \& Pastura, G. (2003). As medicações estimulantes. Em L. A. Rohde \& P. Mattos (Orgs.), Princípios e práticas em TDAH - Transtorno de déficit de atenção/ hiperatividade (pp. 161-173). Porto Alegre: Artmed.

Corrreia Filho, G. A., \& Pastura, G. (2003). As Medicações Estimulantes. Em L. A. Rohde, \& P. Mattos, P. (Orgs.), Princípios e práticas em TDAH (pp. 35-52). Porto Alegre: Artmed.

Falcomata, T. S., Northup, J. A., Dut, A., Stricker, J. M., Vinquist, K. M., \& Engebretos, B. J. (2008). A preliminary analysis of instructional control in the maintenance of appropriate behavior. Journal of Applied Behavior Analysis, 41 (3), 429-434.

Faraone, S. V., Biedreman, J. M. D., Weber, W. B. A., \& Russell, R. L. B. A. (1998). Psychiatric, Neuropsychological, and Psychosocial Features of DSM-IV Subtypes of AttentionDeficit/Hyperactivity Disorder: Results From a Clinically Referred Sample. Journal of the American Academy of Child \& Adolescent Psychiatry, 37(2), 185-193.

Faraone, S. V., Perlis, R. H., Doyle, A. E., Somller, J. W., Goralnick, J., Holmgren, M. A., \& Skar, P. (2005). Molecular Genetics of Attention-Deficit/Hyperactivity Disorder, Biological Psychiatry, 57(11), $1313-1323$.
Freire, A.C. C., \& Pondé, M. P. (2005). Estudo Piloto da prevalência do Transtorno de Déficit de Atenção e Hiperatividade entre crianças escolares na cidade de Salvador, Bahia, Brasil. Arquivos de Neuropsiquiatria, 63 2B, 474-478.

Goldiamond, I. (2002). Toward a constructional approach to social problems: ethical and constitutional issues raised by applied behavior analysis. Behavior and Social Issues, 11, 108-197.

Goldman, L. S., Genel, M., Bezman, R. J., \& Slanetz, P. J. (1998). Diagnosis and Treatment of Attention-Deficit/Hyperactivity Disorder in Children and Adolescents. The Journal of the American Medical Association, 279, 1100-1107.

Guardiola, A., Terra, A. R., Ferreira, L. T. C., \& Londero, R. G. (1999). Uso de Amitriptilina na Síndrome de Hiperativiade com Déficit de Atenção. Arquivo de Neuropsiquiatria, 57 (3A), 599-605.

Heger, S., Trott, G. E., Meusers, M., Schulz, E., Rothenberger, A., Rettig, K., Medor, R., Schreiner, A., Remxchmidt, H. (2006). Switching from a short-acting to a long-acting methylphenidate preparation: a multicentre, open study in children with ADHD. Z. Kinder Jugendpsychiatry Psychother, 34 (4), 257-265.

Jessen, P. S. (2001). ADHD comorbidity and treatment outcomesin the MTA [introduction to the special section]. Journal of the American Academy of Child and Adolescent Psychiatry, 40 (2), 134-136.

Jessen, P. S., Hinshaw, S. P., \& Kraemer, H. C. (2001). ADHD comorbidity findings from the MTA study: comparing comorbid subgroups. Journal of American Academy of Child and Adolescent Psychiatry, 40 (2), 147-158.

Johnston, C., \& Mash, E. J. (2001). Families of children with attention-deficit/hyperactivity disorder: review and recommendations for future research. Clinical Child and Family Psychology Review, 4 (3), 183-207.

Kazdin, A. E., \& Kagan, J. (1994). Models of dysfunction in developmental psychopathology. Clinical Psychology, Science and Practice, 1, 35-52.

Kennedy, C.H., Caruso, M. e Thompson, T. (2001). Experimental analyses of gene-brain-behavior relations some notes on their application. Journal of Applied Analysis of Behavior, 34, 539549.

Kessler, R. C., Chiu, W. T., Demler, O., Merikangas, K. R., \& Walters, E. E. (2005). Prevalence, severity, and comorbidity of 12-month DSM-IV disorders in the national comorbidity survey replication. Archives of General Psychiatry, 62, 617-627.

Klorman, R. (1991). Cognitive event-related potentials in attention deficit disorder. Journal of Learning Disabilities, 24, 130-140.

Knapp, P., Johannpeter, J., Lyszkowski, L., \& Rohde, L. A . (2002). Terapia Cognitivo-Comportamental no TDAH. Em L. A. Rohde \& P. Mattos. (Orgs.), Princípios e práticas em TDAH (pp. 183198). Porto Alegre: Artes Médicas.

Kodak, T., Northup, J., \& Kelley, M. E. (2007). An evaluation of the types of attention that maintain problem behavior. Journal of Applied Behavior Analysis, 40 (1), 167-171.

Kooij, J. J., Buiterlaar, J. K., Van Den Oord, E. J., Furer, J. W., Rijnders, C. A. T., \& Hodiamont, P. G. (2005). Internal and external validity of Attention-Deficit Hyperactivity Disorder in a population-based sample of adults. Psychological Medicine, 35 (6), 817-827. 
Lousier, B. J., McGranth, P. G., \& Klein, R. M. (1996). Error patterns on the continuous performance test in non-medicated and medicated samples of children with and without ADAHD? A meta-analytic review. Journal of Psychology and Psychiatry, 37, 971-987.

Louzã Neto, M. R (2010). TDAH transtorno de déficit de atenção/ hiperatividade ao longo da vida. Porto Alegre: Artimed.

Mattos, P. (2005). No mundo da lua: perguntas e respostas sobre o transtorno do déficit de atenção com hiperatividade em crianças, adolescentes e adultos. 4ed. São Paulo: Lemos.

Michelson, D., Faries, D., Wernicke, J., Kelsey, D., Kendrick, K., Sallee, F. R., \& Spencer, T. (2001). Atomoxetine in the treatment of children and adolescents with attention-deficit/hyperactivity disorder: a randomized, placebo-controlled, dose-response study. Pediatrics, 108 (5), 1-9.

Millstein, R. B., Wilens, T. E., Biederman, J., \& Spencer, T. J. (1997). Presenting ADHD symptoms and subtypes in clinically referred adults with ADHD. Journal of Attention Disorders, 2 (3), 159-166.

Murphy, K., Barkley, R. A., \& Bush, T. M. A. (2002). Young adults with attention deficit hyperactivity disorder: subtype differences in comorbidity, educational and clinical history. Journal of Nervous \& Mental Disease, 190 (3), 147-157.

Naves, A. R. C. X. (2008). Contingências e metacontingências familiares: um Estudo exploratório. Dissertação de Mestrado, Universidade de Brasília, Brasília.

Offord, D. R., Boyle, M. H., Racine, Y. A., Fleming, J. E., Cadman, D T., Blum, H. M., Byrne, C. R.N., Links, P., Lipman, E., Macmillan, H. L. Era, G., Naomi, I., Sanford, M., Szatmari, P., Thomas, H., Woodward, C. (1992). Outcome, Prognosis, and Risk in a Longitudinal Follow-up Study. Journal of the American Academy of Child \& Adolescent Psychiatry, 31 (5), 976.

Organização Mundial de Saúde (1993). Classificação de transtornos mentais e de comportamentos da CID-10: descrições clínicas e diretrizes diagnósticas. Porto Alegre: Artmed.

Pastor, P. N., \& Reuben, C. A(2002). Attention Deficit Disorder and Learning Disability? United States, 1997-98. Vital and health statistics, series 10(206) 1-12.

Phelan, T. W. (2005). Transtorno de Déficit de Atenção e Hiperativade. M. Books do Brasil Ltda: São Paulo.

Polanchzyk, G., Zeni C., Genro, J. P., Roman, T., Hutz, M.H., Rohde, L.A. (2005). Attention-deficit/hiperactivity disorder: advancing on pharmacogenomics. Pharmacogenocmics, 6, 225-234.

Rohde L. A. (2002). ADHD in Brazil: the DSM-IV criteria in a culturally different population. Journal of the American Academy of Child and Adolescent Psychiatry, 159, 36-42.

Rohde L. A., Busnello, E. A., Chachamovich E., Vieira G. M., Pinzon V. \& Ketzer C. R. (1998). Transtorno de déficit de atenção/hiperatividade: revisando conhecimentos. Revista ABP-APAL, 20 (4), 166-178.

Rohde L. A., Mattos, P. \& col. (2003). Principios e práticas em $T D A H$. Porto Alegre: Artmed.

Rohde, L. A., \& Halpern, R. (2004). Transtorno de déficit de atenção/ hiperatividade: atualizado. Journal de Pediatria, 80(2), 61-70.

Rohde, L. A., Zeni, C., Polanczyk, G., \& Hutz, M. (2004a). New insights on Attention-Deficit/Hyperactivity Disorder Pharmacogenimics. Drug Development Research, 51, 1-8.
Rohde, L. A., \& Benxzik, E. B. P. (1999) Transtorno de déficit de atenção /Hiperatividade: O que é ? Como ajudar? Porto Alegre: Artmed.

Rohde, L. A., Barbosa, G., Tramontina, S., \& Polanxzyk, G. (2000). Transtorno de déficit de atenção/hipatividade. Revista Brasileira de Psiquiatria. 22, (Suplemento II), 7-11.

Rohde, L. A., Biederman, J., Busnello, E. A., Zimmermann, H., Schmitz, M., Martins \& Tramotina, S. (1999). ADAH in a school sample of Brazilian adolescents: a study of prevalence, comorbid conditions, and impairments. Journal American Academic Children Adolescence Psychiatry, 38 (6), 716-722.

Rohde, L. A., Cosntantino, E., Filho, M., Benettti, L., Gallois, C., \& Kieling, C. (2004b). Transtorno de déficit de atenção/ hiperatividade na infância e na adolescência: considerações clínicas e terapêuticas. Revista Psiquiátrica Clínica, 31 (3), 124-131.

Rosales-Ruiz, J. \& Baer, D. M. (1996). A behavior-analytic view of development. Em S. W. Bijou \& E. Ribes (Eds.), New directions in behavior development (pp. 155-180). Reno: Context Press.

Rotta, N. T. (2006). Transtorno da atenção: aspectos clínicos. Em N. T. Rotta, L. Ohlweiler \& R. S. Riesgo (Orgs.), Transtorno da aprendizagem Abordagem Neurobiológica e Multidisciplinar. Porto Alegre: Artmed.

Rowland, A. S., Umbach, D. M., Catoe, K. E., Stallone, L., Long, S., Rabiner, D., Naftel, A. J., Panck, D., Faulk, R., \& Sandler, D. P. (2001). Studying the epidemiology of attention-deficit hyperactivity disorder: screening method and pilot result. Canadian Journal of psychiatry, 46(10), 931-940.

Rutter, M., \& Sroufe, L. A. (2000). Developmental psychopathology. Concepts and challenges. Development and Psychopathology, 12, 265-296.

Satterfield, J. H., \& Dawson, M. E. (1971). Electrodermal correlates of hyperactiviyy in children. Psychophysiology, 8, 191-197.

Schteinschnaider, A., Plaghos, L. L., Gargugino, S., Riveros, D., Lazarowski, A., Intruvini, S., \& Massaro, M. (2000). Cerebral arteritis following methylphenidate use. Journal of Child Neurology, 15(4), 265-267.

Schwartzman, J. S. (2001) Transtorno de déficit de atenção. São Paulo: Memnon/Mackenzie.

Segenreich, D., \& Mattos, P. (2004). Eficácia da bupropiona no tratamento do TDAH. Uma revisão sistemática e análise crítica de evidências. Revista de Psiquiat Clínica, 31(3),117-123

Skinner, B. F. (1957/1978). O comportamento verbal (M. P. Villalobos, Trad.). São Paulo: Cultrix.

Skinner, B.F. (2000). Ciência e comportamento Humano. Tradução de J. C. Todorov e R. Azzi. São Paulo: Martins Fontes. (Trabalho original publicado em 1953).

Smith, C., \& Strick, L. (2001). Dificuldades de Aprendizagem de A $a$ Z. $1^{\mathrm{a}}$ ed. Ed. Artes Médicas.

Sonneville, L. M. J., Njiokiktjien, C., \& Bos, H. (1994). Methilphenidate and information processing. Part 1: differentiation between responders and noresponderes. Part 2: efficacy in responders. Journal of Clinical and Experimental Neuropsychology, 16, 877-897.

Sonuga-Barke, E. J. S. (2002). Psychological heterogeneity in $\mathrm{AD} / \mathrm{HD}$ - a dual pathway model of behaviour and cognition. Behavioural Brain Research, 130, 29-36. 
Souza, I., Serra, M. A., Mattos, P., \& Franco, V. A. (2001). Comorbidade em crianças e adolescentes com transtorno do déficit de atenção. Arquivo Neuropsiquiátrico, 59 (2-B), 4001-406.

Strayhorn, J. M. Jr., (2002). Self-Control: Theory and Research, Journal of the American Academy of Child \& Adolescent Psychiatry, 41(1), 7-16.

Swanson, J., Deutsch, C., Cantwell, D., Posner, M., Kennedy, J. L., Barr, C. L., Moyzis, R., Schuck, S., Flodman, P., Spence, M. A., \& Wasdell, M.. (2001). Genes and attention-deficit hyperactivity disorder. Clinical Neuroscience Research, 1(3), 207-216.

Sykes, D. H., Douglas, V. I., \& Morgenstern (1972). The effect of methylphenidate (Ritalin) on sustained attention in hyperactivity children. Psychopharmacology, 25, 262-274.

Szobot, C. M., \& Stone, I. R. (2003). Transtorno de déficit de atenção/hiperatividade: bases neurológicas. Em L. A. Rohde, P. Mattos, e cols. (Orgs.) Princípios e práticas em Transtorno de Déficit de Atenção/Hiperatividade, (v. 3, pp. 53-62).

Thapar, A., Holmes, J., Poulton, K., \& Harreington, R. (1999). Genetic basis of attention deficit and hyperactivity. The British Journal of Psychiatry, 174, 105-111.

Todd, R. D. (2000). Genetics of Attention Deficit Hyperactivity Disorder: are we ready for molecular genetic studies? American Journal of Medical Genetics (Neuropsychiatric genetics), 36, (3), 241-243.

Vaidya, C. J., Austin, G., Kirkorian, G., Ridlehuber, H. W., Desmond, J. E., Glover, G. H., \& Gabrieli J. D. E. (1998). Selective effects of methylphenidate in attention deficit hyperactivity disorder: A functional magnetic resonance study, Proc Natl Acad Sci U $S$ A, 95(24), 14494-14499.
Van der Meere, J., Boudewijn, G., \& Stemerdink, N. (1996). Changing a response set in normal development and in ADHD children with and without. Journal of Abnormal Child Psychology, 24(6), 1573-2835.

Wilens, T. A. M., Pelham, W. B., Stein, M. C., Conners, C. K. D., Abikoff, H. E., Atkins, M. F., August, G. G., Greenhill, L. H., McBurnett, K. I., Palumbo, D. J., Swanson, J. K., \& Wolraich, M. L. (2003). ADHD treatment with once-daily OROS methylphenidate Interim 12-month results from a long-term open-label study, Journal of the American Academy of Child \& Adolescent Psychiatry, 42, 424-433.

Wilson, H. K., Cox, D. J., Merkel, R. L., Moore, M., \& Coghill, D. (2006). Effect of extended release stimulant-based medications on neuropsychological functioning among adolescents with Attention-Deficit/Hyperactivity Disorder. Archives of Clinical Neuropsychology, 21(8), 797-807.

Zametkin, A. M. D., \& Rapoport, J. M. D. (1987). Neurobiology of Attention Deficit Disorder With Hyperactivity: Where Have We Come in 50 Years? Journal of the American Academy of Child \& Adolescent Psychiatry, 26(5), 676-686.

Recebido em 11.03 .09

Primeira decisão editorial em 19.08.09

Versão final em 03.12.09

Aceito em 24.03.10 